\title{
Analyzing Social Media to Explore the Attitudes and Behaviors Following the Announcement of Successful COVID-19 Vaccine Trials: Infodemiology Study
}

Jean-Christophe Boucher ${ }^{1}$, PhD; Kirsten Cornelson ${ }^{2}$, PhD; Jamie L Benham ${ }^{3,4}$, MD; Madison M Fullerton ${ }^{4}$, MSc; Theresa Tang ${ }^{4}$, BHSc (Hons); Cora Constantinescu ${ }^{5}$, MD; Mehdi Mourali ${ }^{6}, \mathrm{PhD}$; Robert J Oxoby ${ }^{7}, \mathrm{PhD}$; Deborah A Marshall $^{3,4}$, PhD; Hadi Hemmati ${ }^{8}$, PhD; Abbas Badami ${ }^{8}$, BsEng; Jia Hu ${ }^{4}, \mathrm{MD}$; Raynell Lang ${ }^{3}$, MD

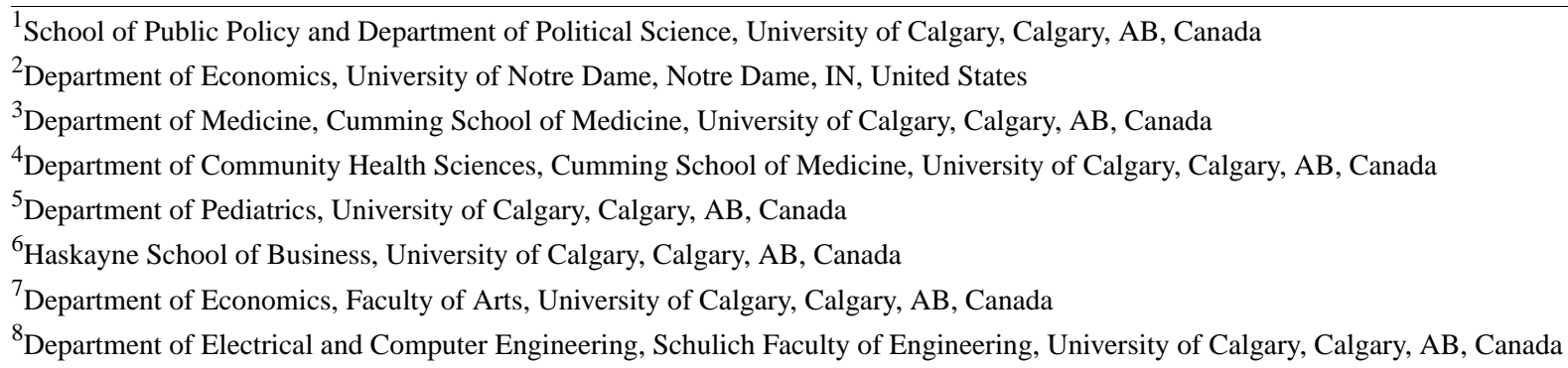

Corresponding Author:

Jean-Christophe Boucher, PhD

School of Public Policy and Department of Political Science

University of Calgary

9068 Ave SW 5th Floor

Calgary, $\mathrm{AB}$

Canada

Phone: 14032208565

Email: jc.boucher@ucalgary.ca

\begin{abstract}
Background: The rollout of COVID-19 vaccines has brought vaccine hesitancy to the forefront in managing this pandemic. COVID-19 vaccine hesitancy is fundamentally different from that of other vaccines due to the new technologies being used, rapid development, and widespread global distribution. Attitudes on vaccines are largely driven by online information, particularly information on social media. The first step toward influencing attitudes about immunization is understanding the current patterns of communication that characterize the immunization debate on social media platforms.
\end{abstract}

Objective: We aimed to evaluate societal attitudes, communication trends, and barriers to COVID-19 vaccine uptake through social media content analysis to inform communication strategies promoting vaccine acceptance.

Methods: Social network analysis (SNA) and unsupervised machine learning were used to characterize COVID-19 vaccine content on Twitter globally. Tweets published in English and French were collected through the Twitter application programming interface between November 19 and 26, 2020, just following the announcement of initial COVID-19 vaccine trials. SNA was used to identify social media clusters expressing mistrustful opinions on COVID-19 vaccination. Based on the SNA results, an unsupervised machine learning approach to natural language processing using a sentence-level algorithm transfer function to detect semantic textual similarity was performed in order to identify the main themes of vaccine hesitancy.

Results: The tweets $(n=636,516)$ identified that the main themes driving the vaccine hesitancy conversation were concerns of safety, efficacy, and freedom, and mistrust in institutions (either the government or multinational corporations). A main theme was the safety and efficacy of mRNA technology and side effects. The conversation around efficacy was that vaccines were unlikely to completely rid the population of COVID-19, polymerase chain reaction testing is flawed, and there is no indication of long-term T-cell immunity for COVID-19. Nearly one-third (45,628/146,191, 31.2\%) of the conversations on COVID-19 vaccine hesitancy clusters expressed concerns for freedom or mistrust of institutions (either the government or multinational corporations) and nearly a quarter $(34,756 / 146,191,23.8 \%)$ expressed criticism toward the government's handling of the pandemic. 
Conclusions: Social media content analysis combined with social network analysis provides insights into the themes of the vaccination conversation on Twitter. The themes of safety, efficacy, and trust in institutions will need to be considered, as targeted outreach programs and intervention strategies are deployed on Twitter to improve the uptake of COVID-19 vaccination.

(JMIR Infodemiology 2021;1(1):e28800) doi: $\underline{10.2196 / 28800}$

\section{KEYWORDS}

coronavirus; COVID-19; public health; social media; Twitter; behavior; risk reduction; attitudes; social network analysis; machine learning

\section{Introduction}

The COVID-19 pandemic is emerging as one of the greatest public health threats in history, with over 140 million infections and 3 million deaths worldwide attributed to the SARS-CoV-2 virus as of April 2021 [1]. As transmission of COVID-19 continues around the globe, a COVID-19 vaccine is an important and valuable tool to reduce the spread of infection. Due to the critical need, the speed of vaccine development, production, and mass rollout has been faster than ever seen before, leading to concerns about vaccine efficacy and safety $[2,3]$. The intricacy surrounding COVID-19 vaccine hesitancy appears to be further reaching and fundamentally different than other immunizations [2]. Vaccine production cannot meet demand, requiring rollout plans for targeted subpopulations, and there are several types of COVID-19 vaccines being used within one country [2]. This has led to increased concern, mistrust, and confusion surrounding COVID-19 vaccination.

Despite the massive undertaking of vaccine development, a vaccination program is only as successful as its uptake. Vaccine hesitancy, defined as a delay in acceptance or refusal of vaccines despite their availability [4], was mentioned by the World Health Organization as one of the top 10 threats to public health in 2019 [5]. The effective rollout of a COVID-19 vaccine strategy may be obstructed by the beliefs and attitudes of vaccine-hesitant individuals worldwide [6]. A recent survey of US adults in April 2020 found that $23 \%$ of persons would not be willing to get vaccinated against COVID-19 [7]. In Canada, a survey in March 2021 demonstrated that $76.9 \%$ of Canadians were very or somewhat willing to receive a COVID-19 vaccine [8].

Vaccine hesitancy is a "multifaceted, deeply complex construct that may be rooted in the moral composition that guides our daily decision making" [8,9]. Studies have identified that vaccination decisions are shaped by multiple complex interactions between individual, community, cultural, historical, political, and societal factors $[2,10]$. There have been several metrics and scales developed and used to measure vaccine confidence and hesitancy [11-13]. The Vaccine Confidence Index (VCI) survey tool was developed to measure individuals' perceptions on the safety, importance, effectiveness, and religious beliefs toward vaccines, which were identified as key drivers of public confidence in vaccination [11]. Another validated score measuring the psychological antecedents of vaccination is known as the $5 \mathrm{C}$ scale [13]. The 5C scale includes confidence (trust and attitudes), complacency (not perceiving diseases as high risk), constraints (structural and psychological barriers), collective responsibility (willingness to protect others), and calculation (information searching) [13].
Negative beliefs about vaccines may prevent the implementation of provaccination policies. Public health officials need to prioritize implementing strategies to help reduce these negative perceptions [14]. Traditional approaches to promoting immunization have assumed that inadequate knowledge of the associated risks and benefits drive hesitancy; however, this stance has proven to be ineffective as an intervention [15]. The source of the hesitancy is often both from lack of information and from lack of trust in institutions such as the government, physicians, and pharmaceutical companies $[16,17]$. Rather than just trying to enhance knowledge, a different approach to overcoming vaccine hesitancy for COVID-19 may be to focus on changing personal attitudes [15].

Prior studies have demonstrated that social media can help understand attitudes and behaviors during public health crises and promote health messaging [18-20]. Particularly, Twitter has been used for public health research and more specifically regarding COVID-19 [18,21]. A recent study aimed to characterize the main topics of Twitter conversations related to COVID-19 and identified four main themes including the origin of the virus, its sources, the impact on people/countries/economy, and lastly methods of mitigating the risk of infection [18].

Attitudes toward vaccination are, in large part, shaped by information and ideas individuals encounter through social media $[22,23]$. Social media is a principal informational forum for vaccination uptake with large proportions of content involving antivaccination messaging [22,24]. Reliable and accurate information on social media is often mixed with inaccurate, conspiratorial, incomplete, or biased messages [22]. A recent study evaluating vaccine hesitancy through content analysis of tweets in Canada identified major themes including safety, suspicion of economic or political motivation, knowledge deficit, opinions of authority figures, and lack of liability from pharmaceutical companies [25]. This study demonstrates the significant utility of using Twitter to better understand vaccine hesitancy at a population level [25]. However, a broader reaching and deeper understanding of current patterns of communication that characterize the immunization debate on social media platforms is needed to inform public health interventions aimed toward influencing attitudes on immunization [15,17,22,23].

This study used social network analysis (SNA) and unsupervised machine learning to characterize COVID-19 vaccine content on Twitter, which allows researchers to access the application programming interface (API). The use of machine learning in social sciences is expanding and has generated interesting methodological conversations on different approaches to study 
COVID-19 sentiment, attitude, and emotion [26-28]. With this study, we aimed to use social media content analysis to provide a further understanding of societal attitudes, communication trends, and barriers to COVID-19 vaccine uptake, at a critical time during the COVID-19 pandemic, just following reporting of initial vaccination trials. We hypothesize that these data will be critical globally for developing targeted outreach programs and intervention strategies on Twitter to impact COVID-19 vaccine hesitancy.

\section{Methods}

\section{Study Data}

Tweets published in English and French using specific words ("COVID" AND ("vacc" OR "vax" OR "immu")) in either the content of the tweet or hashtag derivatives were collected through the Twitter API between November 19 and 26, 2020. This Boolean query was selected in order to maximize inclusivity without adding unnecessary noise to our data set. For example, with the query "vacc," we effectively targeted all derivatives such as "vaccine," "vaccines," "vaccinated," "vaccination," "vaccinations," and associated hashtags. Furthermore, it follows search queries from similar studies examining vaccine hesitancy and social media [29-31]. English and French were selected as they are two of the most common languages used on social media. The data set included several features, such as descriptive information about the user, username, content of tweets (hashtags, relationship among users such as retweet, replies, and mentions, etc), self-reported location of the user, number of followers, date of account creation, and time of tweet posting. Tweets were extracted using Twitter's public streaming API allowing researchers to collect a random sample of tweets in real time of up to $1 \%$ of all public tweets published daily.

This time period was crucial in COVID-19 vaccination conversations on social media as it came a week after pharmaceutical companies (Pfizer and Moderna) announced successful trials of their COVID-19 vaccines [32,33]. For the first time since the beginning of the COVID-19 pandemic in early 2020, social media conversations on vaccination were based, at least in part, on plausible empirical information about the efficacy and availability of a vaccine. We therefore wanted to evaluate the public's initial reaction and response to COVID-19 vaccination. Few prior studies have included this time frame during the analysis period [30,34-36].

\section{Social Network Analysis}

In this study, we designed a data analytics workflow by first using SNA to identify social media accounts most likely expressing doubtful or mistrustful opinions on COVID-19 vaccination. This network was created using a weighted retweet directed network to represent connections between accounts. Although retweets are not a perfect indicator of like-mindedness, on aggregate, users have a proclivity to engage more with accounts that reflect some form of social or intellectual homophily [37]. Through SNA, we examined the underlying structure of community clustering within the broader social network exposed by online interactions, isolating different "communities" of like-minded users. The Louvain modularity method [38] was used to detect subclusters of online communities mentioning COVID-19 vaccination.

\section{Natural Language Programming Analysis}

Based on our SNA results, we developed an unsupervised machine learning approach to natural language processing by using a sentence-level algorithm transfer function to detect semantic textual similarity [15]. Our goal was to examine how antivaccine conversation clusters talk about or frame a possible COVID-19 vaccine without prior assumptions about the nature of the conversation. For this analysis, we first tokenized our sentences (tweets) and cleaned the data set, removing duplicates, stop words, symbols, numbers, punctuation, URLs, whitespaces, and stemmed words to their roots. We also added a language identifier to remove tweets in languages other than French or English and reduce noise in the data set. We then used DistilBERT [39], a knowledge distillation learning model, for sentence-level embedding. DistilBERT is a compressed version of BERT (Bidirectional Encoder Representations from Transformers), which retains much of the computational accuracy of BERT without the environmental costs associated with high-dimensionality embeddings. DistilBERT positions all sentences (here tweets from the antivaccine conversation) in a multidimensionality vector space from which we can compare the semantic similarity of tweets.

We used an agglomerative hierarchical cluster model to identify a relevant number of clusters from the multidimensionality output produced by the DistilBERT computation, which provided us a measure to identify different topics of similar tweets. To infer topics from our clustering modeling, we used a bi-gram of term frequency-inverse document frequency (tf-idf), which measures the originality of a word by comparing the number of occurrences of the word in a document (term frequency) and the number of documents with the word (inverse document frequency). This measure allows us to undervalue words that appear frequently in most documents (such as "the") and provide little information, and overvalue words that appear sporadically in the corpus, but often in some documents. Topics were then inferred manually based on the cluster model and informed by the tf-idf output. Figure 1 illustrates our data analytics pipeline. 
Figure 1. Data analytics workflow. NLP: natural language processing.

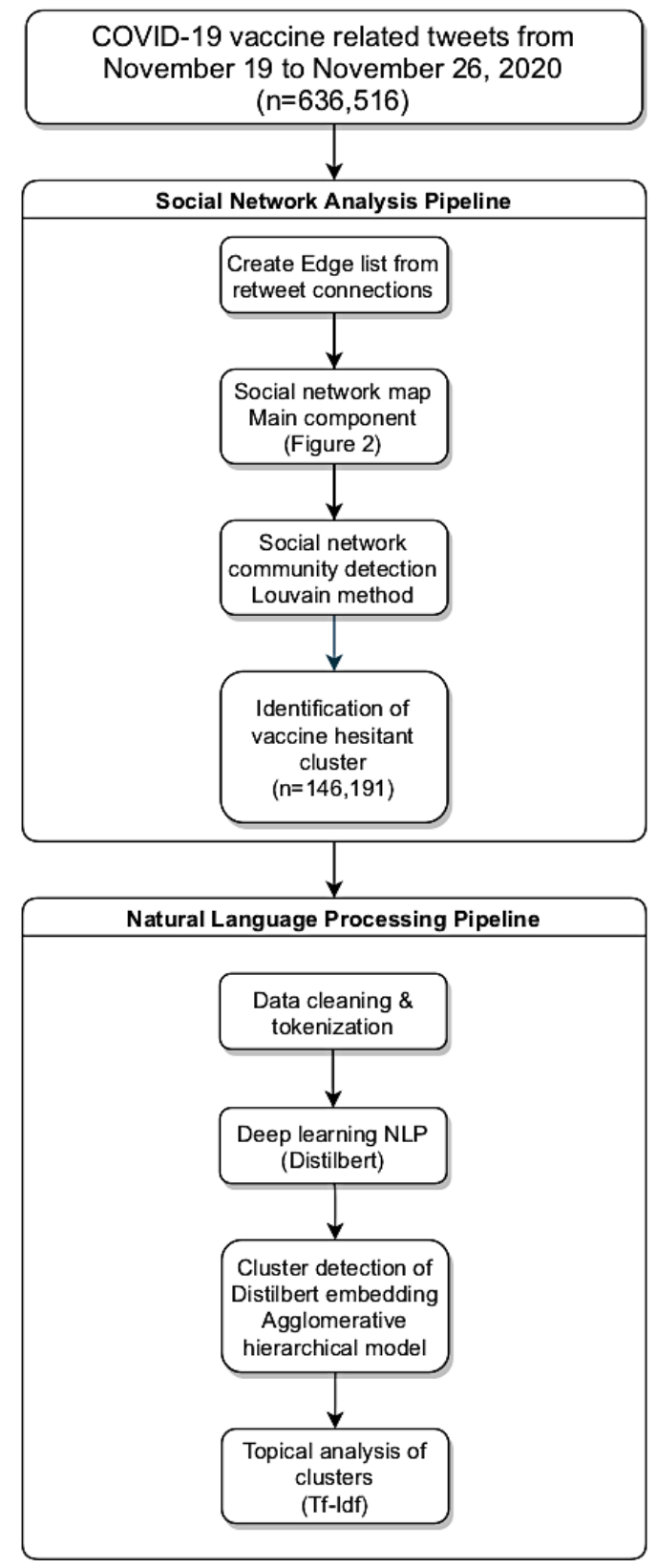

\section{Results}

In total, 636,516 tweets were collected from 428,535 accounts, for an average of 79,564 tweets per day. Figure 2 presents the cluster map of COVID-19 immunization conversations on Twitter between November 19 and November 26, 2020. We found a polarized conversation about immunization on social media.

During this observation period, a large proportion of accounts debating COVID-19 immunization on Twitter were connected and exposed to social media conversations promoting vaccination narratives. The largest cluster (green), comprising approximately $49.4 \%(n=211,549)$ of Twitter accounts, revealed a vaccine acceptant point of view. Based on degree centrality, the cluster seemed to overlap with more progressive-leaning political leaders, such as Hillary Clinton and US Representative Alexandria Ocasio-Cortez (D-14-NY), and mainstream news media, such as the NY Post, the Hill, ABC, and Reuters. A second provaccine cluster (orange) centered on the Indian COVID-19 immunization online debate, with political leaders, such as Rahul Gandhi and Press Trust of India (the largest news agency in India), leading the conversation.

As Figure 2 indicates, we also found two clusters opposite to these vaccine acceptant clusters exhibiting more vaccine hesitant narratives. There were $23.4 \%(n=146,191)$ of conversations on Twitter during this period of observation that can be directly attributed to vaccine hesitancy. First, in red, our study identified a large cluster comprising 88,892 Twitter handles accounting for $18.4 \%$ of all accounts in the cluster. These interactions from the Twittersphere in English originated mostly from the United States, the United Kingdom, and Canada, and gravitated around accounts of prominent antivaccine physicians and organizations, right-wing activists, show hosts, such as Rush Limbaugh in the United States and Simon Dolan or Michael J Blair in the United Kingdom, and some alternative news organizations, such as 
Breitbart News. In this conversation cluster, we found an overlap between ideologically leaning advocates, especially those associated at the margins of right-wing or Conservative parties, and antivaccine online conversations. We found considerable cross-pollination between accounts originating from all across the English Twittersphere, demonstrating the internationalization of COVID-19 vaccination conversations on social media. Second, in blue, a smaller cluster representing $2.7 \%(n=11,509)$ of accounts appeared to be shaped around Francophone (from France and Québec) vaccine hesitant conversations.

Figure 2. Twitter retweet cluster of COVID-19 vaccination (November 19 to November 26, 2020). The vaccine acceptant cluster ( $\mathrm{n}=211,549)$, vaccine hesitant cluster $(n=88,892)$, Indian vaccine acceptant cluster $(n=28,713)$, and French vaccine hesitant cluster ( $=11,509)$ are seen. Nodes represent specific Twitter accounts, while edges represent retweet activity between accounts. Presented are the four largest online communities mentioning COVID-19 vaccination.

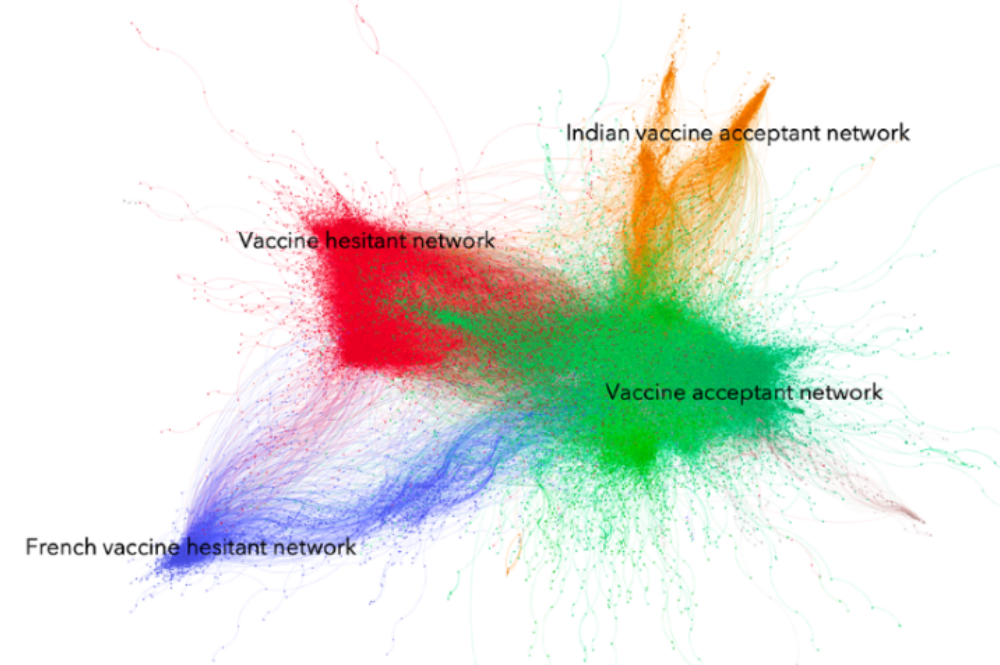

Examining the COVID-19 vaccine hesitancy narrative during this observation period provided key insights on what drives attitudes. As shown in Table 1, our unsupervised machine learning analysis identified 12 specific archetypical vaccine hesitancy tweets (we tested a different number of clusters between 9 to 20). Social media content highlighted concerns over the efficacy and the safety of a possible COVID-19 vaccine fitting into the $5 \mathrm{C}$ scale domain of confidence. Five broad categories focused on vaccine efficacy in our data set. The first topic (topic 3 in Table 1) regrouped tweets suggesting that attempts to produce a COVID-19 vaccine, especially using mRNA technology, remain tentative. These tweets highlighted our failure to develop an HIV vaccine with mRNA and that existing mRNA vaccines for COVID-19 have not been tested enough to demonstrate efficacy. The second category of vaccine hesitancy tweets concentrating on efficacy (topic 2) focused on a comment made by Sir John Irving Bell on November 20, 2020, that existing vaccines were unlikely to completely rid the population of COVID-19. The third and fourth vaccine efficacy topics (topics 7 and 8) grouped together tweets from prominent physicians arguing that existing polymerase chain reaction testing is flawed and that there are no indications of long-term T-cell immunity for COVID-19. The last topic highlighted (topic 10), which obtained subsequent media attention, suggested that as much as $25 \%$ of the population would have contracted COVID-19 by the time a vaccine would be rolled out, and consequently, the vaccine would be unnecessary to reach herd immunity.
As for the concern of vaccine safety, we found two broad groups of tweets. The first (topic 5) highlighted a classic antivaccine story where an emergency medical technician/fire rescue was required to get TDAP (tetanus, diphtheria, and pertussis) boosters and had complications. Although not specifically addressing the issue of a COVID-19 vaccine, these tweets emphasize how existing antivaccine narratives have created a baseline from which some individuals frame a COVID-19 vaccine. The second topic (topic 9) centered on tweets in French framing a COVID-19 vaccine as a poison and suggesting that mRNA technology has not been tested yet and would be harmful.

Although safety and efficacy concerns remain a major component explaining COVID-19 vaccine hesitancy, it is only half of the story. As we examined the content of tweets of users integrated in the vaccine hesitancy online cluster, we found a large proportion of those interactions that emphasized a concern for personal freedom and/or some form of mistrust of institutions. In the 5C scale of psychological antecedents of vaccination, confidence in vaccines includes trust in the system that delivers the vaccines and the motivation of policy makers who decide on the need of vaccines [13]. Tweets were framed in three directions. First, a large percentage of such tweets $(n=45,628,31.2 \%)$ expressed some criticism toward the government's handling of the COVID-19 crisis, especially decisions to curb individual freedom. COVID-19 immunization is framed as the right of individuals to decide for themselves whether or not to be vaccinated. Any indication that governmental authorities might require vaccination is perceived 
as a direct attempt to limit individual freedom. Two main topics (topics 1 and 12) highlighted mistrust in government policy, with one condemning the Johnson government in the United Kingdom for proposing that individuals vaccinated for COVID-19 could receive "freedom passes" and one criticizing Denmark's decision to cull its mink population to halt the spread of a coronavirus variant. Some topics (topics 6 and 11) expressed mistrust in multinational corporations, most notably airline companies, such a Qantas, who suggested that vaccination should be made compulsory for international travel. This decision is framed as a direct restriction of personal freedom by multinational corporations, and some users made a direct reference to populist narratives suggesting that these policies would only affect commercial flights and elites would be able to avoid vaccination while using private flights.

Table 1. Inferred topic analysis of the COVID-19 vaccination hesitancy cluster (November 19 to November 26, 2020).

\begin{tabular}{llll}
\hline Topic & Topic keywords bi-gram (tf-idf) & Inferred topic & Tweets (N=146,191), $\mathrm{n}(\%)$ \\
\hline 1 & $\begin{array}{l}\text { Countries test, results multinational, multinational companies, the } \\
\text { Johnson, commie, passes two, proposing freedom, commie proposing, } \\
\text { full commie, Johnson government, gone full, government gone }\end{array}$ & Trust in the government & $33,578(23.0 \%)$
\end{tabular}
most disturbing, bell talking, professor sir, irving bell, john irving, irving, sir john

3 Produced cell, unlicensed produced, lines aborted, shots wont, HIV/AIDS, won't walk, test enough, infection enough, antibodies past, enough effective, enough antibodies, past infection Presi, COVID literally, one two, presi im, corner presi, literaly around, im sitting, sitting thinking, thinking incredible, incredible one nick healthy, jer, emtfire, gau nick, jer gau, damage jer, dayprofessor sir, tweet dayprofessor, dayprofessor

Passports we, elite continue, the elite, commercial flights, fly private, breaking qantas, ceo confirms, you've vaccinated, compulsory international, international, confirms proof, proof you've

$7 \quad$ Attacked, attacked since, since apr, apr highlighting, highlighting imp, viciously, imp tcell, sarscov, despite published, published COVID, viciously attacked, ive viciously

Label new, staff form, group label, jabs poison, longterm tcell, immunity cases, watch interview, interview dr, science longterm, discussing hysteria, phd discussing, dr phd.

9 Arn caca, quand, faire, arn, bonjour, confinement vaccin, avec, caca confinement, vaccin contre, contre le, le vaccin

10 Coronavirus im, pr quarter, newspapers, im delighted, delighted mainstream, mainstream newspapers, newspapers picking, picking pr.

11 Fly must, want fly, begins if, must take

12 Protestors, force in, could force, COVID law, authorities could, Denmark, proposed forced, protesting proposed, protestors protesting, in Denmark, Denmark protestors, law authorities

\author{
COVID-19 vaccine hesitancy: Efficacy 28,818 (19.7\%)
}

$11,631(8.0 \%)$

Support for Trump's
the COVID-19 crisis

COVID-19 vaccine hesitancy: Side ef- 9579 (6.6\%) fects

Trust in multinational corporations

$9286(6.4 \%)$

COVID-19 vaccine hesitancy: Efficacy 7896 (5.4\%)

COVID-19 vaccine hesitancy: Efficacy 6921 (4.7\%)

COVID-19 vaccine hesitancy: Side ef- 2822 (1.9\%) fects

COVID-19 vaccine hesitancy: Efficacy 2320 (1.6\%)

Trust in multinational corporations

$1586(1.1 \%)$

Trust in the government

$1178(0.8 \%)$
A final residual category (topic 4) in the vaccine hesitancy conversation singled out a tweet by radio host Rush Limbaugh mentioning that two COVID-19 vaccines were now approved and praising President Trump's management of the crisis. This tweet was widely circulated in the vaccine hesitant conversation cluster and underlines the reality that any conversation on COVID-19 immunization, and to some degree, vaccine hesitancy clusters on social media, intersect with broader clusters structured by current political polarization.

Figure 3 presents the approximate distribution of these inferred topics in COVID-19 vaccine hesitancy clusters identified during this observation period and allows us to understand what shapes an individual's attitude toward a COVID-19 vaccine. First, as this data set was collected right after the news of positive trials of COVID-19 vaccines by Pfizer and Moderna, more than half of the tweets $(n=76,531,52.4 \%)$ mentioned vaccine efficacy and raised suspicions on whether creating vaccines using mRNA technology was achievable or whether immunization would be long-lasting. With respect to vaccine safety, only $8.5 \%$ $(n=12,401)$ of the social media debate in vaccine hesitancy clusters doubted its safety. Second, nearly one-third ( $n=45,628$, $31.2 \%$ ) of conversations on vaccine hesitancy clusters on Twitter expressed concerns for freedom or mistrust of institutions (either the government or multinational corporations). These results suggest that one key determinant of vaccine hesitancy is trust in institutions. It suggests that vaccine confidence building is a problem of shaping attitudes toward COVID-19 vaccines, which falls into the realm of health policy, as well as promoting social/political trust, which falls more into the realm of politics. 
Figure 3. Distribution of inferred topics in COVID-19 vaccine hesitancy clusters on social media. This figure presents the aggregated results of inferred topics in vaccine hesitancy clusters from Table 1.

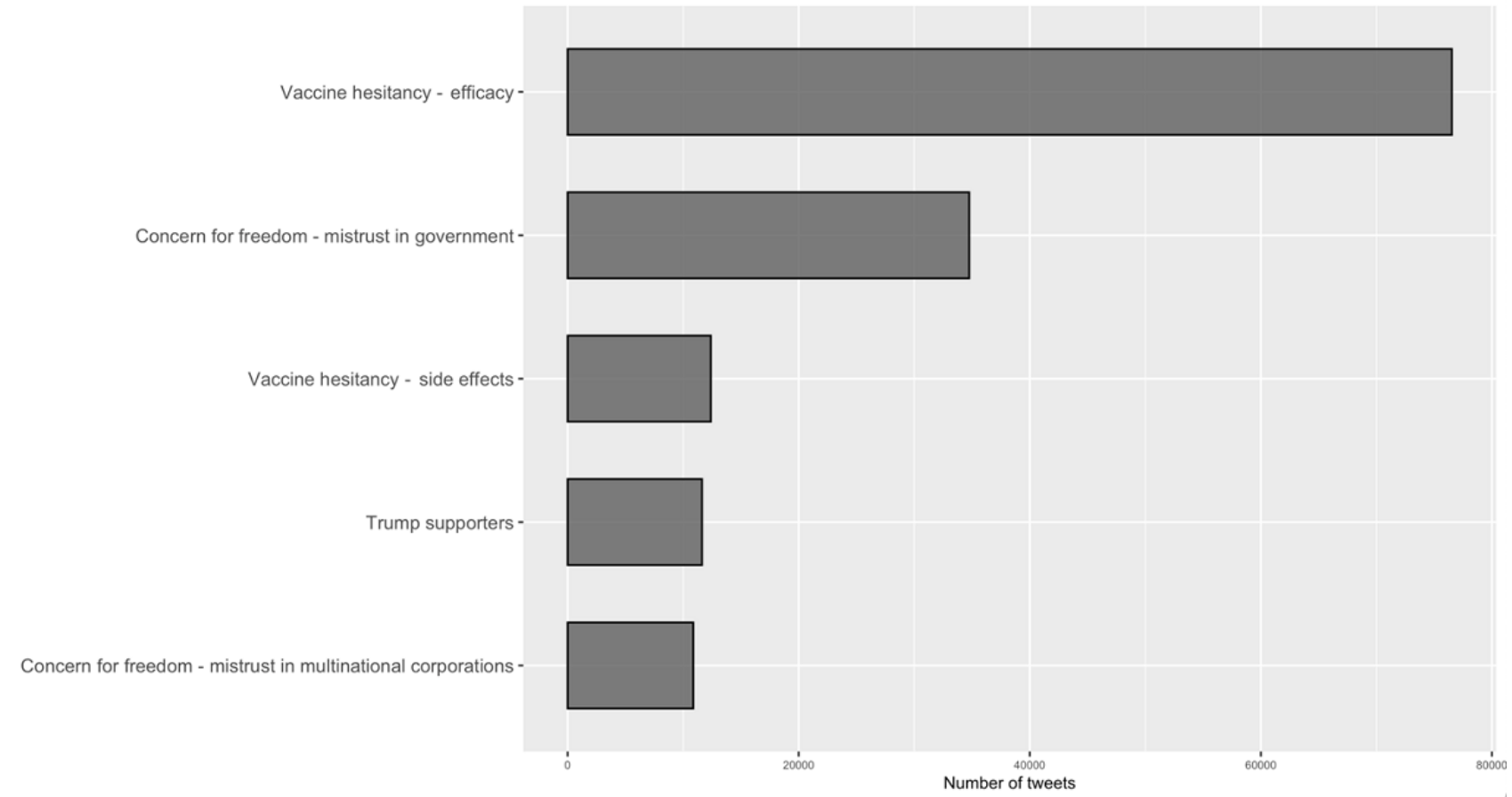

\section{Discussion}

\section{Principal Findings}

We analyzed more than 600,000 tweets over 1 week just following the announcement of successful COVID-19 vaccine trials, to characterize and understand global public perceptions and attitudes surrounding the COVID-19 vaccine and themes driving vaccine hesitancy. Our analysis revealed contrasting conversations about COVID-19 immunization on social media with both vaccine acceptant and vaccine hesitant clusters. Identified were the main themes driving the vaccine hesitant conversation at that time, including concerns of safety, efficacy, and freedom, and mistrust in institutions (either the government or multinational corporations). A main theme was the safety and efficacy of mRNA technology and side effects. The conversation around efficacy was that vaccines were unlikely to completely rid the population of COVID-19, polymerase chain reaction testing is flawed, and there is no indication of long-term T-cell immunity for COVID-19. Nearly one-third (31.2\%) of the conversations on COVID-19 vaccine hesitancy clusters expressed concerns for freedom or mistrust of institutions (either the government or multinational corporations) and nearly a quarter $(23.8 \%)$ expressed criticism toward the government's handling of the pandemic.

The main themes identified in this study fall under the domain of confidence using the $5 \mathrm{C}$ scale. Confidence is a measure of a person's level of trust in vaccine safety and efficacy, as well as in those involved in vaccine administration including policy makers and health professionals. Studies have shown that confidence is positively correlated with attitudes toward vaccination, knowledge of vaccination, and trust in health care, while it is negatively correlated with conspiracy mentality and medical harms [13]
The speed of development, production, and mass rollout of a COVID-19 vaccine has been unprecedented and has led to concerns around the safety and efficacy of vaccination. A common theme identified at that time was concern regarding mRNA technology. mRNA-based therapeutics have been used for cancer vaccines in the past; however, compared to other vaccine technology, it has not been clinically tested to the same extent [40]. Dror et al published results of a survey in which $70 \%$ of the general public responded with concerns about the safety of the COVID-19 vaccine [41]. We identified that less than $10 \%$ of the social media debate in vaccine hesitancy conversations during our observation period doubted its safety. Nevertheless, as COVID-19 vaccines are rolled out, it is highly probable that antivaccine social media conversations will transition from arguing about efficacy to questioning vaccine safety.

Mistrust in institutions has emerged as a predominant theme of vaccine hesitancy conversations on Twitter. Prior literature has reported mistrust in doctors, government sources, and pharmaceutical companies as reasons for hesitancy [16]. Governments are directly involved in many aspects of vaccine development, from funding to eventual safety approval. Individuals who believe the government is incompetent or malicious may not trust that these functions have been carried out in an appropriate way. Trust in government covaries strongly with generalized trust in other people and feelings of connectedness to others in society [42-48]. These measures of "social capital" in turn have been linked with reduced willingness to contribute to public good [43]. For example, there is a link between government trust and willingness to pay taxes [49]. Conversely, increased ethnic or political fragmentation, which creates feelings of division in society, has been shown to reduce the quality of the government and reduce physical distancing during the COVID-19 pandemic [50,51]. Because 
the COVID-19 vaccine has public benefits that go beyond individual protection, individuals with low social trust may be less willing to contribute to the public good by getting the vaccine. Furthermore, recent studies using survey data have been increasingly associating trust in the government with COVID-19 behavior and vaccine hesitancy, and our social media data add to this literature [52,53].

There is extensive literature examining both individual and aggregate correlates of trust. At the individual level, trust is positively correlated with education [54] and civic engagement [42]. Aggregate measures of social trust vary with the actual performance of the government, and poor economic growth, high crime, mass protests, and political scandals appear to reduce the trust of citizens in the government $[55,56]$. Conversely, increasing transparency in the government appears to improve public trust in authorities [57]. Highlighted is the importance of building trust in institutions, which needs to be incorporated into models aimed at targeting vaccine hesitancy in addition to the traditional pillars of communication, information, and cognition.

Globally, persons are challenged with an overabundance of information on COVID-19 and COVID-19 vaccination, in which misinformation has been disseminated rampantly, likely fueling hesitancy [58]. Yaqub et al highlighted in a critical review of vaccine hesitancy that "hesitancy is not a rare phenomenon or confined solely to antivaccinationists; it includes people who have not yet rejected vaccination. Focusing on only vaccine uptake rates and neglecting underlying attitudes is likely to underestimate the challenge of maintaining vaccination coverage in the future" [16]. We demonstrated that social media analysis provides insights into societal attitudes, communication trends, and barriers to vaccine uptake that must be considered when developing strategies to address vaccine hesitancy.

The strength of this study lies in the methodology undertaken, which involved a bottom-up approach for the identification of cases and SNA. Previous studies have generally adopted a top-down approach to data collection, isolating known antivaccine accounts and analyzing its content and diffusion. Although critical in understanding the structure and nature of antivaccination framing on social media, such methods run the risk of selection bias. Moreover, by analyzing tweets in both French and English, we were able to broaden the scope of our vaccine conversation clusters, increasing the generalizability of this work. The noise of this analysis was minimized by linking the vaccination keywords with "COVID."

\section{Limitations}

There are several limitations in our work. Although social media is increasingly used as a source of information and social interaction, even on matters related to health policy, it is an environment where participants self-select themselves in the population and is not a representative sample of the general population. In this sense, our results reflect a specific conversation around COVID-19 vaccination, and studies focusing on other social media platforms (eg, Facebook, WhatsApp, Reddit, and YouTube) and more traditional news media would offer a more complete overview of how antivaccine narratives are structured. Demographic segmentation of these clusters was not possible as further background information on the individuals in each cluster was not available; however, this would be a valuable component to future research. Our analysis was done on data collected right at the onset of news confirming the successful clinical trials of COVID-19 vaccines by Pfizer and Moderna. Our research offers a baseline from which we can understand the evolution of the online debate about COVID-19 vaccination. However, our assumption is that such a conversation will evolve and change throughout the pandemic, and we should expect the saliency of antivaccine arguments (safety, efficacy, and trust in institutions) to fluctuate as new information and policies are put in place. Future research is needed to monitor the COVID-19 vaccine hesitancy conversation through adopting a dynamic approach by collecting tweets over longer time periods and analyzing the patterns of change over time. Finally, as data were collected through the Twitter streaming API, the sample we analyzed may not have been fully randomized. The Twitter streaming API tends to overrepresent central users and is influenced by Twitter's sampling algorithm. Furthermore, given the size of the data set, we could not remove bots, which may have potentially skewed certain results.

\section{Conclusions}

The recent global rollout of COVID-19 vaccination has brought vaccine hesitancy to the forefront in managing this pandemic. Hesitancy in accepting COVID-19 vaccination is fundamentally different from other vaccinations due to the new technologies being used, rapid development, and widespread global distribution. Attitudes on vaccines are largely driven by online information, particularly information on social media. We demonstrated that social media content and network analysis provides insights into societal attitudes, communication trends, and barriers to vaccine uptake. Identified themes driving the vaccine hesitant conversation included concerns of safety, efficacy, and freedom, and mistrust in institutions (either the government or multinational corporations). These themes will need to be considered as targeted outreach programs and intervention strategies are deployed globally in attempts to change personal attitudes on Twitter and improve the uptake of COVID-19 vaccination.

\section{Acknowledgments}

We would like to thank Mohammad Jafar Mashhadi and Emad Jabbar for their contribution to the data analysis. This study was funded by a COVID-19 Rapid Response Funding Grant from Alberta Innovates (grant 202100489). 


\section{Authors' Contributions}

JCB, RL, KC, JLB, TT, MM, MMF, CC, DAM, RJO, HH, and JH were involved in initial concepts, literature searches, and funding applications. JCB and $\mathrm{AB}$ obtained all data and performed all analyses. JCB, RL, and KC wrote the initial draft of the paper. All authors made edits and contributions to the final draft of this manuscript.

\section{Conflicts of Interest}

None declared.

\section{References}

1. Coronavirus Resource Center. Johns Hopkins University \& Medicine. URL: https://coronavirus.jhu.edu/ [accessed 2021-03-12]

2. Dubé E, MacDonald NE. How can a global pandemic affect vaccine hesitancy? Expert Rev Vaccines 2020 Oct;19(10):899-901. [doi: 10.1080/14760584.2020.1825944] [Medline: 32945213]

3. Lurie N, Saville M, Hatchett R, Halton J. Developing Covid-19 Vaccines at Pandemic Speed. N Engl J Med 2020 May 21;382(21):1969-1973. [doi: 10.1056/NEJMp2005630] [Medline: 32227757]

4. Report of the SAGE Working Group on Vaccine Hesitancy. World Health Organization. 2014. URL: http://www.who.int/ immunization/sage/meetings/2014/october/SAGE working group revised report vaccine hesitancy.pdf [accessed 2021-08-02]

5. Ten Threats to Global Health in 2019. World Health Organization. URL: https://www.who.int/news-room/spotlight/ ten-threats-to-global-health-in-2019 [accessed 2020-10-05]

6. Schaffer DeRoo S, Pudalov NJ, Fu LY. Planning for a COVID-19 Vaccination Program. JAMA 2020 Jun 23;323(24):2458-2459. [doi: 10.1001/jama.2020.8711] [Medline: 32421155]

7. Trujillo K, Motta M. A majority of vaccine skeptics plan to refuse a COVID-19 vaccine, a study suggests, and that could be a big problem. The Conversation. 2020. URL: https://theconversation.com/ a-majority-of-vaccine-skeptics-plan-to-refuse-a-covid-19-vaccine-a-study-suggests-and-that-could-be-a-big-problem-137559 [accessed 2020-10-06]

8. COVID-19 vaccine willingness among Canadian population groups. Statistics Canada. 2021. URL: https://www150. statcan.gc.ca/n1/pub/45-28-0001/2021001/article/00011-eng.htm [accessed 2021-08-02]

9. McAteer J, Yildirim I, Chahroudi A. The VACCINES Act: Deciphering Vaccine Hesitancy in the Time of COVID-19. Clin Infect Dis 2020 Jul 28;71(15):703-705 [FREE Full text] [doi: 10.1093/cid/ciaa433] [Medline: $\underline{32282038}$ ]

10. Dubé E, Gagnon D, MacDonald N, Bocquier A, Peretti-Watel P, Verger P. Underlying factors impacting vaccine hesitancy in high income countries: a review of qualitative studies. Expert Rev Vaccines 2018 Nov;17(11):989-1004. [doi: 10.1080/14760584.2018.1541406] [Medline: 30359151]

11. de Figueiredo A, Simas C, Karafillakis E, Paterson P, Larson HJ. Mapping global trends in vaccine confidence and investigating barriers to vaccine uptake: a large-scale retrospective temporal modelling study. The Lancet 2020 Sep 26;396(10255):898-908 [FREE Full text] [doi: 10.1016/S0140-6736(20)31558-0] [Medline: 32919524]

12. Shapiro GK, Tatar O, Dube E, Amsel R, Knauper B, Naz A, et al. The vaccine hesitancy scale: Psychometric properties and validation. Vaccine 2018 Jan 29;36(5):660-667. [doi: 10.1016/j.vaccine.2017.12.043] [Medline: 29289384]

13. Betsch C, Schmid P, Heinemeier D, Korn L, Holtmann C, Böhm R. Beyond confidence: Development of a measure assessing the 5C psychological antecedents of vaccination. PLoS One 2018;13(12):e0208601 [FREE Full text] [doi: 10.1371/journal.pone.0208601] [Medline: 30532274]

14. Stecula DA, Kuru O, Albarracin D, Jamieson KH. Policy Views and Negative Beliefs About Vaccines in the United States, 2019. Am J Public Health 2020 Oct;110(10):1561-1563. [doi: 10.2105/AJPH.2020.305828] [Medline: 32816542]

15. Du J, Luo C, Shegog R, Bian J, Cunningham RM, Boom JA, et al. Use of Deep Learning to Analyze Social Media Discussions About the Human Papillomavirus Vaccine. JAMA Netw Open 2020 Nov 02;3(11):e2022025 [FREE Full text] [doi: 10.1001/jamanetworkopen.2020.22025] [Medline: 33185676]

16. Yaqub O, Castle-Clarke S, Sevdalis N, Chataway J. Attitudes to vaccination: a critical review. Soc Sci Med 2014 Jul;112:1-11 [FREE Full text] [doi: 10.1016/j.socscimed.2014.04.018] [Medline: 24788111]

17. Loomba S, de Figueiredo A, Piatek SJ, de Graaf K, Larson HJ. Measuring the impact of COVID-19 vaccine misinformation on vaccination intent in the UK and USA. Nat Hum Behav 2021 Mar;5(3):337-348. [doi: 10.1038/s41562-021-01056-1] [Medline: $\underline{33547453}$ ]

18. Abd-Alrazaq A, Alhuwail D, Househ M, Hamdi M, Shah Z. Top Concerns of Tweeters During the COVID-19 Pandemic: Infoveillance Study. J Med Internet Res 2020 Apr 21;22(4):e19016 [FREE Full text] [doi: 10.2196/19016] [Medline: 32287039]

19. Steffens MS, Dunn AG, Wiley KE, Leask J. How organisations promoting vaccination respond to misinformation on social media: a qualitative investigation. BMC Public Health 2019 Oct 23;19(1):1348 [FREE Full text] [doi: 10.1186/s12889-019-7659-3] [Medline: 31640660] 
20. Sinnenberg L, Buttenheim AM, Padrez K, Mancheno C, Ungar L, Merchant RM. Twitter as a Tool for Health Research: A Systematic Review. Am J Public Health 2017 Jan;107(1):e1-e8. [doi: 10.2105/AJPH.2016.303512] [Medline: 27854532]

21. Tsao S, Chen H, Tisseverasinghe T, Yang Y, Li L, Butt ZA. What social media told us in the time of COVID-19: a scoping review. The Lancet Digital Health 2021 Mar;3(3):e175-e194 [FREE Full text] [doi: 10.1016/S2589-7500(20)30315-0] [Medline: 33518503]

22. Wilson SL, Wiysonge C. Social media and vaccine hesitancy. BMJ Glob Health 2020 Oct;5(10):e004206 [FREE Full text] [doi: 10.1136/bmjgh-2020-004206] [Medline: 33097547]

23. Tavoschi L, Quattrone F, D'Andrea E, Ducange P, Vabanesi M, Marcelloni F, et al. Twitter as a sentinel tool to monitor public opinion on vaccination: an opinion mining analysis from September 2016 to August 2017 in Italy. Hum Vaccin Immunother 2020 May 03;16(5):1062-1069 [FREE Full text] [doi: 10.1080/21645515.2020.1714311] [Medline: 32118519 ]

24. Johnson NF, Velásquez N, Restrepo NJ, Leahy R, Gabriel N, El Oud S, et al. The online competition between pro- and anti-vaccination views. Nature 2020 Jun;582(7811):230-233. [doi: 10.1038/s41586-020-2281-1] [Medline: $\underline{32499650]}$

25. Griffith J, Marani H, Monkman H. COVID-19 Vaccine Hesitancy in Canada: Content Analysis of Tweets Using the Theoretical Domains Framework. J Med Internet Res 2021 Apr 13;23(4):e26874 [FREE Full text] [doi: 10.2196/26874] [Medline: $\underline{33769946}$ ]

26. Lucas C, Nielsen RA, Roberts ME, Stewart BM, Storer A, Tingley D. Computer-Assisted Text Analysis for Comparative Politics. Polit. anal 2017 Jan 04;23(2):254-277. [doi: 10.1093/pan/mpu019]

27. Greene D, Cross JP. Exploring the Political Agenda of the European Parliament Using a Dynamic Topic Modeling Approach. Polit. Anal 2017 Mar 13;25(1):77-94. [doi: 10.1017/pan.2016.7]

28. Hung M, Lauren E, Hon ES, Birmingham WC, Xu J, Su S, et al. Social Network Analysis of COVID-19 Sentiments: Application of Artificial Intelligence. J Med Internet Res 2020 Aug 18;22(8):e22590 [FREE Full text] [doi: 10.2196/22590] [Medline: 32750001]

29. Himelboim I, Xiao X, Lee DKL, Wang MY, Borah P. A Social Networks Approach to Understanding Vaccine Conversations on Twitter: Network Clusters, Sentiment, and Certainty in HPV Social Networks. Health Commun 2020 May;35(5):607-615. [doi: 10.1080/10410236.2019.1573446] [Medline: 31199698]

30. Hussain A, Tahir A, Hussain Z, Sheikh Z, Gogate M, Dashtipour K, et al. Artificial Intelligence-Enabled Analysis of Public Attitudes on Facebook and Twitter Toward COVID-19 Vaccines in the United Kingdom and the United States: Observational Study. J Med Internet Res 2021 Apr 05;23(4):e26627 [FREE Full text] [doi: 10.2196/26627] [Medline: 33724919]

31. Luo X, Zimet G, Shah S. A natural language processing framework to analyse the opinions on HPV vaccination reflected in twitter over 10 years (2008 - 2017). Hum Vaccin Immunother 2019;15(7-8):1496-1504 [FREE Full text] [doi: 10.1080/21645515.2019.1627821] [Medline: 31194609]

32. Polack FP, Thomas SJ, Kitchin N, Absalon J, Gurtman A, Lockhart S, C4591001 Clinical Trial Group. Safety and Efficacy of the BNT162b2 mRNA Covid-19 Vaccine. N Engl J Med 2020 Dec 31;383(27):2603-2615 [FREE Full text] [doi: 10.1056/NEJMoa2034577] [Medline: 33301246]

33. Jackson LA, Anderson EJ, Rouphael NG, Roberts PC, Makhene M, Coler RN, mRNA-1273 Study Group. An mRNA Vaccine against SARS-CoV-2 - Preliminary Report. N Engl J Med 2020 Nov 12;383(20):1920-1931 [FREE Full text] [doi: 10.1056/NEJMoa2022483] [Medline: 32663912]

34. Lyu JC, Han EL, Luli GK. COVID-19 Vaccine-Related Discussion on Twitter: Topic Modeling and Sentiment Analysis. J Med Internet Res 2021 Jun 29;23(6):e24435 [FREE Full text] [doi: 10.2196/24435] [Medline: 34115608]

35. Liu S, Liu J. Understanding Behavioral Intentions Toward COVID-19 Vaccines: Theory-Based Content Analysis of Tweets. J Med Internet Res 2021 May 12;23(5):e28118 [FREE Full text] [doi: 10.2196/28118] [Medline: 33939625]

36. Allington D, McAndrew S, Moxham-Hall V, Duffy B. Coronavirus conspiracy suspicions, general vaccine attitudes, trust and coronavirus information source as predictors of vaccine hesitancy among UK residents during the COVID-19 pandemic. Psychol Med 2021 Apr 12:1-12 [FREE Full text] [doi: 10.1017/S0033291721001434] [Medline: $\underline{33843509]}$

37. Yang Z, Guo J, Cai K, Tang J, Li J, Zhang L, et al. Understanding retweeting behaviors in social networks. In: CIKM '10: Proceedings of the 19th ACM International Conference on Information and Knowledge Management. 2010 Presented at: 19th ACM International Conference on Information Knowledge Management; October 26-30, 2010; Toronto, ON, Canada p. 1633-1636. [doi: 10.1145/1871437.1871691]

38. Blondel VD, Guillaume J, Lambiotte R, Lefebvre E. Fast unfolding of communities in large networks. J. Stat. Mech 2008 Oct 09;2008(10):P10008. [doi: 10.1088/1742-5468/2008/10/P10008]

39. Sanh V, Debut L, Chaumond J, Wolf T. DistilBERT, a distilled version of BERT: smaller, faster, cheaper and lighter. arXiv. 2020. URL: https://arxiv.org/abs/1910.01108 [accessed 2021-08-05]

40. Abbasi J. COVID-19 and mRNA Vaccines-First Large Test for a New Approach. JAMA 2020 Sep 22;324(12):1125-1127. [doi: 10.1001/jama.2020.16866] [Medline: 32880613]

41. Dror AA, Eisenbach N, Taiber S, Morozov NG, Mizrachi M, Zigron A, et al. Vaccine hesitancy: the next challenge in the fight against COVID-19. Eur J Epidemiol 2020 Aug;35(8):775-779. [doi: 10.1007/s10654-020-00671-y] [Medline: 32785815]

42. Keele L. Social Capital and the Dynamics of Trust in Government. Am J Political Science 2007 Apr;51(2):241-254. [doi: 10.1111/j.1540-5907.2007.00248.x]

43. Putnam R. Bowling Alone: The Collapse and Revival of American Community. New York, NY: Simon \& Schuster; 2000. 
44. Nannestad P. What Have We Learned About Generalized Trust, If Anything? Annu. Rev. Polit. Sci 2008 Jun;11(1):413-436. [doi: 10.1146/annurev.polisci.11.060606.135412]

45. Rothstein B, Stolle D. The State and Social Capital: An Institutional Theory of Generalized Trust. Comp Politics 2008 Jul 01;40(4):441-459. [doi: 10.5129/001041508x12911362383354]

46. Knack S, Keefer P. Does Social Capital Have an Economic Payoff? A Cross-Country Investigation. The Quarterly Journal of Economics 1997 Nov 01;112(4):1251-1288. [doi: 10.1162/003355300555475]

47. Knack S. Social Capital and the Quality of Government: Evidence from the States. American Journal of Political Science 2002 Oct;46(4):772-785. [doi: 10.2307/3088433]

48. Jennings MK, Stoker L. Social Trust and Civic Engagement across Time and Generations. Acta Polit 2004 Dec 13;39(4):342-379. [doi: 10.1057/palgrave.ap.5500077]

49. Antinyan A, Corazzini L, Pavesi F. Does trust in the government matter for whistleblowing on tax evaders? Survey and experimental evidence. Journal of Economic Behavior \& Organization 2020 Mar;171:77-95. [doi: 10.1016/j.jebo.2020.01.014]

50. Alesina A, Zhuravskaya E. Segregation and the Quality of Government in a Cross Section of Countries. American Economic Review 2011 Aug 01;101(5):1872-1911. [doi: 10.1257/aer.101.5.1872]

51. Algan Y, Hémet C, Laitin DD. The Social Effects of Ethnic Diversity at the Local Level: A Natural Experiment with Exogenous Residential Allocation. Journal of Political Economy 2016 Jun;124(3):696-733. [doi: 10.1086/686010]

52. Merkley E, Loewen PJ. Anti-intellectualism and the mass public's response to the COVID-19 pandemic. Nat Hum Behav 2021 Jun 28;5(6):706-715. [doi: 10.1038/s41562-021-01112-w] [Medline: 33911228]

53. Lazarus JV, Ratzan SC, Palayew A, Gostin LO, Larson HJ, Rabin K, et al. A global survey of potential acceptance of a COVID-19 vaccine. Nat Med 2021 Feb;27(2):225-228 [FREE Full text] [doi: 10.1038/s41591-020-1124-9] [Medline: 33082575]

54. Brehm J, Rahn W. Individual-Level Evidence for the Causes and Consequences of Social Capital. American Journal of Political Science 1997 Jul;41(3):999-1023. [doi: 10.2307/2111684]

55. Chanley VA, Rudolph TJ, Rahn WM. The origins and consequences of public trust in government: a time series analysis. Public Opin Q 2000;64(3):239-256. [doi: 10.1086/317987] [Medline: 11114267]

56. Sangnier M, Zylberberg Y. Protests and trust in the state: Evidence from African countries. Journal of Public Economics 2017 Aug;152:55-67. [doi: 10.1016/j.jpubeco.2017.05.005]

57. Buell RW, Norton MI. Surfacing the Submerged State with Operational Transparency in Government Services. SSRN Journal 2013:1-24. [doi: 10.2139/ssrn.2349801]

58. How to report misinformation online. World Health Organization. URL: https://tinyurl.com/3mh9bpp2 [accessed 2021-08-02]

\section{Abbreviations}

API: application programing interface

BERT: Bidirectional Encoder Representations from Transformers

SNA: social network analysis

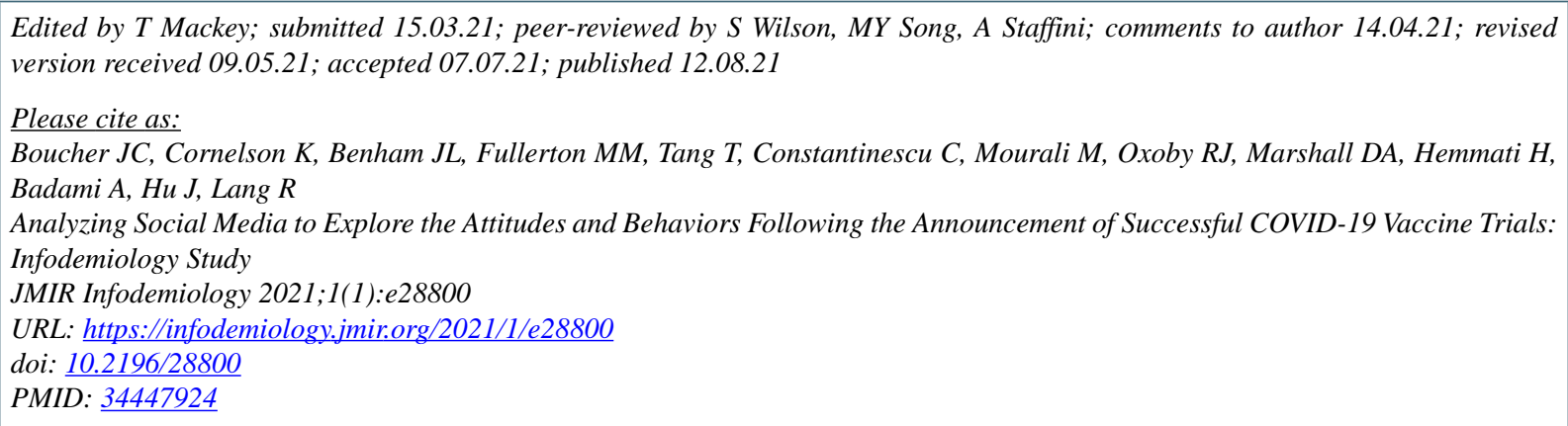

(CJean-Christophe Boucher, Kirsten Cornelson, Jamie L Benham, Madison M Fullerton, Theresa Tang, Cora Constantinescu, Mehdi Mourali, Robert J Oxoby, Deborah A Marshall, Hadi Hemmati, Abbas Badami, Jia Hu, Raynell Lang. Originally published in JMIR Infodemiology (https://infodemiology.jmir.org), 12.08.2021. This is an open-access article distributed under the terms of the Creative Commons Attribution License (https://creativecommons.org/licenses/by/4.0/), which permits unrestricted use, distribution, and reproduction in any medium, provided the original work, first published in JMIR Infodemiology, is properly cited. The complete bibliographic information, a link to the original publication on https://infodemiology.jmir.org/, as well as this copyright and license information must be included. 